\title{
SOME COMMON FIXED POINT THEOREM IN METRIC SPACE USING INTEGRAL TYPE MAPPINGS
}

\author{
RAMAKANT BHARDWAJ \\ Department of Mathematics, Truba Instt. Of Engineering \& Information Technology Bhopal, India
}

\begin{abstract}
In this paper we prove some fixed point theorems for self mapping satisfying a contractive condition of integral type in complete metric spaces. Our results are version of some known results .

Key words:- Fixed point, Common Fixed point, complete metric space, Continuous Mapping, Compatible Mappings.

AMS Subject Classification: - 47H10, 54H25.

\section{INTRODUCTION AND PRELIMINARIES}

Impact of fixed point theory in different branches of mathematics and its applications is immense. The first result on fixed points for contractive type mapping was the much celebrated Banach's contraction principle by S. Banach [1] in 1922. In the general setting of complete metric space, this theorem runs as the follows,

Theorem 1.1 (Banach's contraction principle)

Let $(X, d)$ be a complete metric space, $c \in(0,1)$ and $f: X \rightarrow X$ be a mapping such that for each $x, y \in X$, $\mathrm{d}(\mathrm{fx}, \mathrm{fy}) \leq \mathrm{cd}(\mathrm{x}, \mathrm{y})$

Then $\mathrm{f}$ has a unique fixed point $\mathrm{a} \in \mathrm{X}$, such that for each $\mathrm{x} \in \mathrm{X}, \lim _{\mathrm{n} \rightarrow \infty} \mathrm{f}^{\mathrm{n}} \mathrm{x}=\mathrm{a}$.

After the classical result, Kannan 5[] gave a subsequently new contractive mapping to prove the fixed point theorem. Since then a number of mathematicians have been worked on fixed point theory dealing with mappings satisfying various type of contractive conditions.

In 2002, A. Branciari [2] analyzed the existence of fixed point for mapping $f$ defined on a complete metric space (X, d) satisfying a general contractive condition of integral type.

\section{Theorem 1.2 (Branciari)}

Let $(X, d)$ be a complete metric space, $c \in(0,1)$ and let $f: X \rightarrow X$ be a mapping such that for each $\mathrm{x}, \mathrm{y} \in \mathrm{X}$,

$$
\int_{0}^{\mathrm{d}(\mathrm{fx}, \mathrm{fy})} \xi(\mathrm{t}) \mathrm{dt} \leq \mathrm{c} \int_{0}^{\mathrm{d}(\mathrm{x}, \mathrm{y})} \xi(\mathrm{t}) \mathrm{dt}
$$

Where $\xi:[0,+\infty) \rightarrow[0,+\infty)$ is a Lesbesgue integrable mapping which is summable on each compact subset of $[0,+\infty)$, non negative, and such that for each $\epsilon>0, \int_{0}^{\epsilon} \xi(\mathrm{t}) \mathrm{dt}$, then $\mathrm{f}$ has a unique fixed point $\mathrm{a} \in \mathrm{X}$ such that for each $\mathrm{x} \in \mathrm{X}, \lim _{\mathrm{n} \rightarrow \infty} \mathrm{f}^{\mathrm{n}} \mathrm{x}=\mathrm{a}$.

After the paper of Branciari, a lot of a research works have been carried out on generalizing contractive conditions of integral type for a different contractive mappings satisfying various known properties. A fine work has been done by Rhoades [6] extending the result of Brianciari by replacing the condition [1.2] by the following

$$
\int_{0}^{d(f x, f y)} \xi(t) d t \leq \int_{0}^{\max }\left\{d(x, y), d(x, f x), d(y, f y), \frac{d(x, f y)+d(y, f x)}{2}\right\} \xi(t) d t
$$

The aim of this paper is to generalize some mixed type of contractive conditions to the mapping and then a pair of mappings, satisfying a general contractive mappings such as Kannan type [3], Chatrterjee type [5], Zamfirescu type [11], etc.

\section{MAIN RESULTS}

Theorem 2.1 Let $\mathrm{f}$ be a self mapping of a complete metric space(X, d), satisfying the following condition:

$$
\begin{array}{r}
\int_{0}^{d(f x, f y)} \xi(t) d t \leq \alpha \int_{0}^{[d(x, f x)+d(y, f y)]} \xi(t) d t+\beta \int_{0}^{[d(x, f y)+d(y, f x)]} \xi(t) d t \\
+\gamma \int_{0}^{\max \{d(x, f y), d(y, f x)\}} \xi(t) d t+\delta \int_{0}^{d(x, y)} \xi(t) d t
\end{array}
$$

For each $\mathrm{x}, \mathrm{y} \in \mathrm{X}$ with non negative reals $\alpha, \beta, \gamma, \delta$ such that $0<2 \alpha+2 \beta+2 \gamma+\delta<1$, where $\xi: \mathcal{R}^{+} \rightarrow \mathcal{R}^{+}$is a lesbesgue- integrable mapping which is summable on each compact subset of $\mathcal{R}^{+}$, non negative, and such that

$$
\text { for each } \epsilon>0, \int_{0}^{\epsilon} \xi(\mathrm{t}) \mathrm{dt}
$$

Then $f$ has a unique fixed point $\mathrm{z} \in \mathrm{X}$ and for each $\mathrm{x} \in \mathrm{X}, \lim _{\mathrm{n} \rightarrow \infty} \mathrm{f}^{\mathrm{n}} \mathrm{x}=\mathrm{z}$.

Proof

For any arbitrary $x_{0} \in X$, we define a sequence $\left\{x_{n}\right\}$ of element of $X$, such that,

$$
\mathrm{x}_{\mathrm{n}+1}=\mathrm{fx}_{\mathrm{n}} \quad \text { for } \mathrm{n}=0,1,2,3, \ldots \ldots \ldots \ldots
$$




\section{RAMAKANT BHARDWAJ / IOSR Journal of Engineering (IOSRJEN)}

Www.iosrjen.org

ISSN : 2250-3021

Vol. 2 Issue 2, Feb.2012, pp. 187-190

Now,

From 2.1,

$$
\int_{0}^{d\left(x_{n}+1, x_{n}+2\right)} \xi(t) d t=\int_{0}^{d\left(f_{n}, x_{n}+1\right)} \xi(t) d t
$$

$$
\begin{aligned}
& \int_{0}^{d\left(\mathrm{fx}_{\mathrm{n}}, \mathrm{fx}_{\mathrm{n}+1}\right)} \xi(\mathrm{t}) d \mathrm{t} \leq \alpha \int_{0}^{\left[\mathrm{d}\left(\mathrm{x}_{\mathrm{n}}, \mathrm{fx}_{\mathrm{n}}\right)+\mathrm{d}\left(\mathrm{x}_{\mathrm{n}+1}, \mathrm{fx}_{\mathrm{n}+1}\right)\right]} \xi(\mathrm{t}) d \mathrm{t} \\
& +\beta \int_{0}^{\left[\mathrm{d}\left(\mathrm{x}_{\mathrm{n}}, \mathrm{fx}_{\mathrm{n}+1}\right)+\mathrm{d}\left(\mathrm{x}_{\mathrm{n}+1}, \mathrm{fx}_{\mathrm{n}}\right)\right]} \xi(\mathrm{t}) \mathrm{dt} \\
& +\gamma \int_{0}^{\max \left\{\mathrm{d}\left(\mathrm{x}_{\mathrm{n}}, \mathrm{fx}_{\mathrm{n}+1}\right), \mathrm{d}\left(\mathrm{x}_{\mathrm{n}+1}, \mathrm{fx}_{\mathrm{n}}\right)\right\}} \xi(\mathrm{t}) \mathrm{dt} \\
& +\delta \int_{0}^{\mathrm{d}\left(\mathrm{x}_{\mathrm{n}}, \mathrm{x}_{\mathrm{n}}+1\right)} \xi(\mathrm{t}) \mathrm{dt} \\
& \int_{0}^{d\left(x_{n}+1, x_{n}+2\right)} \xi(t) d t \leq \alpha \int_{0}^{\left[d\left(x_{n}, x_{n+1}\right)+d\left(x_{n}+1, x_{n}+2\right)\right]} \xi(t) d t \\
& +\beta \int_{0}^{\left[\mathrm{d}\left(\mathrm{x}_{\mathrm{n}}, \mathrm{x}_{\mathrm{n}+2}\right)+\mathrm{d}\left(\mathrm{x}_{\mathrm{n}+1}, \mathrm{x}_{\mathrm{n}+1}\right)\right]} \xi(\mathrm{t}) \mathrm{dt} \\
& +\gamma \int_{0}^{\max \left\{\mathrm{d}\left(\mathrm{x}_{\mathrm{n}}, \mathrm{x}_{\mathrm{n}+2}\right), \mathrm{d}\left(\mathrm{x}_{\mathrm{n}+1}, \mathrm{x}_{\mathrm{n}+1}\right)\right\}} \xi(\mathrm{t}) \mathrm{dt}
\end{aligned}
$$

$+\delta \int_{0}^{\mathrm{d}\left(\mathrm{x}_{\mathrm{n}}, \mathrm{x}_{\mathrm{n}+1}\right)} \xi(\mathrm{t}) \mathrm{dt}$

Which implies,

$$
\int_{0}^{d\left(x_{n}+1, x_{n}+2\right)} \xi(t) d t \leq\left(\frac{\alpha+\beta+\gamma+\delta}{1-\alpha-\beta-\gamma}\right) \int_{0}^{d\left(x_{n}, x_{n}+1\right)} \xi(t) d t
$$

Thus by routine calculation,

$$
\int_{0}^{d\left(x_{n}, x_{n}+1\right)} \xi(t) d t \leq h^{n} \int_{0}^{d\left(x_{0}, x_{1}\right)} \xi(t) d t
$$

Where $\mathrm{h}=\left(\frac{\alpha+\beta+\gamma+\delta}{1-\alpha-\beta-\gamma}\right)<1$

Taking limit of (2.4) as $n \rightarrow \infty$, we get

$$
\lim _{n \rightarrow \infty} \int_{0}^{d\left(x_{n}, x_{n}+1\right)} \xi(t) d t=0
$$

We now show that $\left\{\mathrm{x}_{\mathrm{n}}\right\}$ is a Cauchy sequence. Suppose that it is not. Then there exists an $\epsilon>0$ and subsequence $\{\mathrm{m}(\mathrm{p})\}$ and $\{\mathrm{n}(\mathrm{p})\}$ such that $\mathrm{m}(\mathrm{p})<n(\mathrm{p})<m(p+1)$ with

$$
\begin{aligned}
& \text { Now } d\left(x_{m(p)}, x_{n(p)}\right)=\epsilon, \quad d\left(x_{m(p)}, x_{n}(p)-1\right)<\epsilon \\
& d\left(x_{m}(p)-1, x_{n}(p)-1\right)<d\left(x_{m}(p)-1, x_{m}(p)\right)+d\left(x_{m}(p), x_{n(p)-1}\right) \\
& d\left(x_{m}(p)-1, x_{n(p)-1}\right)<d\left(x_{m(p)-1}, x_{m(p)}\right)+\epsilon \\
& \text { Hence } \\
& \lim _{p \rightarrow \infty} \int_{0}^{d\left(x_{m}(p)-1, x_{n}(p)-1\right)} \xi(t) d t=\int_{0}^{\epsilon} \xi(t) d t
\end{aligned}
$$

Using (2.3), (2.6), and (2.8) we get

$$
\int_{0}^{\epsilon} \xi(\mathrm{t}) \mathrm{dt} \leq \int_{0}^{\mathrm{d}\left(\mathrm{x}_{\mathrm{m}}(\mathrm{p}), \mathrm{x}_{\mathrm{n}}(\mathrm{p})\right)} \xi(\mathrm{t}) \mathrm{dt} \leq \mathrm{h} \int_{0}^{\mathrm{d}\left(\mathrm{x}_{\mathrm{m}}(\mathrm{p})-1, \mathrm{x}_{\mathrm{n}}(\mathrm{p})-1\right)} \xi(\mathrm{t}) \mathrm{dt} \leq \mathrm{h} \int_{0}^{\epsilon} \xi(\mathrm{t}) \mathrm{dt}
$$

Which is contradiction, since $h \in(0,1)$. therefore $\left\{x_{n}\right\}$ is a Cauchy, hence converges to $\mathrm{z} \in \mathrm{X}$ from 2.1, we get

$$
\begin{aligned}
& \int_{0}^{d\left(f z, f x_{n}\right)} \xi(t) d t \leq \alpha \int_{0}^{\left[d(z, f z)+d\left(x_{n}, f x_{n}\right)\right]} \xi(t) d t+\beta \int_{0}^{\left[d\left(z, f x_{n}\right)+d\left(x_{n}, f z\right)\right]} \xi(t) d t \\
& +\gamma \int_{0}^{\max \left\{\mathrm{d}\left(\mathrm{z}, \mathrm{fx}_{\mathrm{n}}\right), \mathrm{d}\left(\mathrm{x}_{\mathrm{n}}, \mathrm{fz}\right)\right\}} \xi(\mathrm{t}) \mathrm{dt}+\delta \int_{0}^{\mathrm{d}\left(\mathrm{z}, \mathrm{x}_{\mathrm{n}}\right)} \xi(\mathrm{t}) \mathrm{dt}
\end{aligned}
$$

Taking limit as $\mathrm{n} \rightarrow \infty$, we get

$$
\int_{0}^{\mathrm{d}(\mathrm{fz}, \mathrm{z})} \xi(\mathrm{t}) \mathrm{dt} \leq(\alpha+\beta+\gamma) \int_{0}^{\mathrm{d}(\mathrm{fz}, \mathrm{z})} \xi(\mathrm{t}) \mathrm{dt}
$$

$$
\text { as } 2 \alpha+2 \beta+2 \gamma+\delta<1 \text {, }
$$

$$
\int_{0}^{d(f z, z)} \xi(t) d t=0
$$

Which, from 2.2, implies that $\mathrm{d}(\mathrm{fz}, \mathrm{z})=0$ or $\mathrm{fz}=\mathrm{z}$.

Next suppose that $w$ is another fixed point of $f$, different from $z$, in X i.e $w \neq z$ then from 2.1 , we have

$$
\int_{0}^{\mathrm{d}(\mathrm{w}, \mathrm{z})} \xi(\mathrm{t}) \mathrm{dt} \leq(2 \beta+\gamma+\delta) \int_{0}^{[\mathrm{d}(\mathrm{w}, \mathrm{fz})+\mathrm{d}(\mathrm{z}, \mathrm{fw})]} \xi(\mathrm{t}) \mathrm{dt}
$$

since $2 \beta+\gamma+\delta<1$, This implies that

$$
\int_{0}^{\mathrm{d}(\mathrm{w}, \mathrm{z})} \xi(\mathrm{t}) \mathrm{dt}=0
$$

Which from 2.2, implies that $\mathrm{d}(\mathrm{z}, \mathrm{w})=0$, and so the fixed point is unique.

\section{Remarks.}

i. On setting $\xi(\mathrm{t})=1$ over $\mathcal{R}^{+}$, the contractive condition of integral type transforms into a general contractive condition not involving integrals. 


\section{RAMAKANT BHARDWAJ / IOSR Journal of Engineering (IOSRJEN)}

Vol. 2 Issue 2, Feb.2012, pp. 187-190

ii. From condition 2.1, of integral type, several contractive mappings of integral type can be obtained.

a. $\quad \beta=\gamma=\delta=0$ and $\alpha \in\left(0, \frac{1}{2}\right)$ gives Kannan mapping of integral type.

b. $\alpha=\beta=\delta=0$ and $\gamma \in\left(0, \frac{1}{2}\right)$, gives Chatterjea map of integral type.

c. $\quad \beta=0 \alpha, \gamma \in\left(0, \frac{1}{2}\right), \delta \in(0,1)$, at least one of the following condition hold:

$\mathrm{z}_{1}: \int_{0}^{\mathrm{d}(\mathrm{fx}, \mathrm{fy})} \xi(\mathrm{t}) \mathrm{dt} \leq \alpha \int_{0}^{[\mathrm{d}(\mathrm{x}, \mathrm{fx})+\mathrm{d}(\mathrm{y}, \mathrm{fy})]} \xi(\mathrm{t}) \mathrm{dt}$

$\mathrm{z}_{2}: \int_{0}^{\mathrm{d}(\mathrm{fx}, \mathrm{fy})} \xi(\mathrm{t}) \mathrm{dt} \leq \gamma \int_{0}^{[\mathrm{d}(\mathrm{x}, \mathrm{fy})+\mathrm{d}(\mathrm{x}, \mathrm{fy})]} \xi(\mathrm{t}) \mathrm{dt}$

$\mathrm{z}_{3}: \int_{0}^{\mathrm{d}(\mathrm{fx}, \mathrm{fy})} \xi(\mathrm{t}) \mathrm{dt} \leq \delta \int_{0}^{\mathrm{d}(\mathrm{x}, \mathrm{y})} \xi(\mathrm{t}) \mathrm{dt}$

Gives Zamfirescu mapping of integral type.

Example 2.2 let $\mathrm{X}=[0,1]$ and $\mathrm{d}$ be usual metric with $\mathrm{d}(\mathrm{x}, \mathrm{y})=|\mathrm{x}-\mathrm{y}|$. Clearly $(\mathrm{X}, \mathrm{d})$ is a complete metric space. Let $\mathrm{f}: \mathrm{X} \rightarrow \mathrm{X}$ be given by $\mathrm{fx}=\frac{\mathrm{x}}{2}$ for all $\mathrm{x} \in[0,1]$. Again $\xi: \mathcal{R}^{+} \rightarrow \mathcal{R}^{+}$be given by $\xi(\mathrm{t})=\frac{\mathrm{t}^{2}}{2}$ for all $\mathcal{R}^{+}$. then for each $\epsilon>0$,

$$
\int_{0}^{\epsilon} \xi(\mathrm{t}) \mathrm{dt}=\int_{0}^{\epsilon} \frac{\mathrm{t}^{2}}{2} \mathrm{dt}=\frac{\epsilon^{3}}{6}>0 .
$$

Now taking $\alpha=\beta=\gamma=\frac{1}{16}$ and $\delta=\frac{1}{8}$, one can easily verify that the condition 2.1, of theorem 2.1 is satisfied with $2 \alpha+2 \beta+2 \gamma+\delta<1$ and 0 is, of course, the unique fixed point of $\mathrm{f}$.

Now our next theorem is the extension of the theorem 2.1, for a pair of mappings.

Theorem 2.3 Let $\mathrm{f}$ and be self mapping of a complete metric space $(\mathrm{X}, \mathrm{d})$, satisfying the following condition:

$$
\begin{aligned}
& \int_{0}^{d(f x, g y)} \xi(t) d t \leq \alpha \int_{0}^{[d(x, f x)+d(y, g y)]} \xi(t) d t+\beta \int_{0}^{[d(x, g y)+d(y, f x)]} \xi(t) d t \\
& +\gamma \int_{0}^{\max \{\mathrm{d}(\mathrm{x}, \mathrm{gy}), \mathrm{d}(\mathrm{y}, \mathrm{fx})\}} \xi(\mathrm{t}) \mathrm{dt}+\delta \int_{0}^{\mathrm{d}(\mathrm{x}, \mathrm{y})} \xi(\mathrm{t}) \mathrm{dt} \quad 2.9
\end{aligned}
$$

For each $\mathrm{x}, \mathrm{y} \in \mathrm{X}$ with non negative reals $\alpha, \beta, \gamma, \delta$ such that $0<2 \alpha+2 \beta+2 \gamma+\delta<1$, where $\xi: \mathcal{R}^{+} \rightarrow \mathcal{R}^{+}$is a lesbesgue- integrable mapping which is summable on each compact subset of $\mathcal{R}^{+}$, non negative, and such that

2.10

$$
\text { for each } \epsilon>0, \int_{0}^{\epsilon} \xi(t) d t
$$

Then fand $g$ have a unique fixed point $\mathrm{z} \in \mathrm{X}$ and for each $\mathrm{x} \in \mathrm{X}, \lim _{\mathrm{n} \rightarrow \infty} \mathrm{f}^{\mathrm{n}} \mathrm{x}=\mathrm{z}$.

Proof

For any arbitrary $x_{0} \in X$, we define a sequence $\left\{x_{n}\right\}$ of element of $X$, such that, $\mathrm{x}_{\mathrm{n}+1}=\mathrm{fx}_{\mathrm{n}}$, and $\mathrm{x}_{\mathrm{n}+2}=\mathrm{gx}_{\mathrm{n}+1} \quad$ for $\mathrm{n}=0,1,2,3, \ldots \ldots \ldots \ldots$

Now,

From 2.9,

$$
\int_{0}^{d\left(x_{n}+1, x_{n}+2\right)} \xi(t) d t=\int_{0}^{d\left(f_{n}, g x_{n}+1\right)} \xi(t) d t
$$

$$
\begin{aligned}
& \int_{0}^{d\left(\mathrm{fx}_{n}, \mathrm{gx}_{\mathrm{n}}+1\right)} \xi(\mathrm{t}) d \mathrm{t} \leq \alpha \int_{0}^{\left[\mathrm{d}\left(\mathrm{x}_{\mathrm{n}}, \mathrm{fx}_{\mathrm{n}}\right)+\mathrm{d}\left(\mathrm{x}_{\mathrm{n}+1}, \mathrm{gx_{n } + 1}\right)\right]} \xi(\mathrm{t}) d \mathrm{t} \\
& +\beta \int_{0}^{\left[d\left(x_{n}, g x_{n+1}\right)+d\left(x_{n+1}, f x_{n}\right)\right]} \xi(t) d t \\
& +\gamma \int_{0}^{\max \left\{\mathrm{d}\left(\mathrm{x}_{\mathrm{n}}, \mathrm{gx}_{\mathrm{n}+1}\right), \mathrm{d}\left(\mathrm{x}_{\mathrm{n}+1}, \mathrm{fx}_{\mathrm{n}}\right)\right\}} \xi(\mathrm{t}) \mathrm{dt} \\
& +\delta \int_{0}^{\mathrm{d}\left(\mathrm{x}_{\mathrm{n}}, \mathrm{x}_{\mathrm{n}}+1\right)} \xi(\mathrm{t}) \mathrm{dt} \\
& \int_{0}^{\mathrm{d}\left(\mathrm{x}_{\mathrm{n}+1}, \mathrm{x}_{\mathrm{n}+2}\right)} \xi(\mathrm{t}) \mathrm{dt} \leq \alpha \int_{0}^{\left[\mathrm{d}\left(\mathrm{x}_{\mathrm{n}}, \mathrm{x}_{\mathrm{n}+1}\right)+\mathrm{d}\left(\mathrm{x}_{\mathrm{n}+1}, \mathrm{x}_{\mathrm{n}+2}\right)\right]} \xi(\mathrm{t}) \mathrm{dt} \\
& +\beta \int_{0}^{\left[\mathrm{d}\left(\mathrm{x}_{\mathrm{n}}, \mathrm{x}_{\mathrm{n}+2}\right)+\mathrm{d}\left(\mathrm{x}_{\mathrm{n}+1}, \mathrm{x}_{\mathrm{n}+1}\right)\right]} \xi(\mathrm{t}) \mathrm{dt} \\
& +\gamma \int_{0}^{\max \left\{\mathrm{d}\left(\mathrm{x}_{\mathrm{n}}, \mathrm{x}_{\mathrm{n}+2}\right), \mathrm{d}\left(\mathrm{x}_{\mathrm{n}+1}, \mathrm{x}_{\mathrm{n}+1}\right)\right\}} \xi(\mathrm{t}) \mathrm{dt}
\end{aligned}
$$

$+\delta \int_{0}^{\mathrm{d}\left(\mathrm{x}_{\mathrm{n}}, \mathrm{x}_{\mathrm{n}}+1\right)} \xi(\mathrm{t}) \mathrm{dt}$

Which implies,

$$
\int_{0}^{d\left(x_{n}+1, x_{n}+2\right)} \xi(t) d t \leq\left(\frac{\alpha+\beta+\gamma+\delta}{1-\alpha-\beta-\gamma}\right) \int_{0}^{d\left(x_{n}, x_{n}+1\right)} \xi(t) d t
$$

2.11

Thus by routine calculation,

2.12

$$
\int_{0}^{d\left(x_{n}, x_{n}+1\right)} \xi(t) d t \leq h^{n} \int_{0}^{d\left(x_{0}, x_{1}\right)} \xi(t) d t
$$

Where $\mathrm{h}=\left(\frac{\alpha+\beta+\gamma+\delta}{1-\alpha-\beta-\gamma}\right)<1$

Taking limit of (2.12) as $n \rightarrow \infty$, we get 


\section{RAMAKANT BHARDWAJ / IOSR Journal of Engineering (IOSRJEN)}

Vol. 2 Issue 2, Feb.2012, pp. 187-190

$$
\lim _{n \rightarrow \infty} \int_{0}^{\mathrm{d}\left(\mathrm{x}_{\mathrm{n}}, \mathrm{x}_{\mathrm{n}}+1\right)} \xi(\mathrm{t}) \mathrm{dt}=0
$$

We now show that $\left\{x_{n}\right\}$ is a Cauchy sequence. Suppose that it is not. Then there exists an $\epsilon>0$ and subsequence $\{\mathrm{m}(\mathrm{p})\}$ and $\{\mathrm{n}(\mathrm{p})\}$ such that $\mathrm{m}(\mathrm{p})<n(\mathrm{p})<m(p+1)$ with

\subsection{4}

$$
\mathrm{d}\left(\mathrm{x}_{\mathrm{m}(\mathrm{p})}, \mathrm{x}_{\mathrm{n}(\mathrm{p})}\right) \geq \epsilon, \quad \mathrm{d}\left(\mathrm{x}_{\mathrm{m}(\mathrm{p})}, \mathrm{x}_{\mathrm{n}(\mathrm{p})-1}\right)<\epsilon
$$

Now

$$
\begin{array}{lc}
\qquad d\left(x_{m(p)-1}, x_{n}(p)-1\right)<d\left(x_{m(p)-1}, x_{m}(p)\right)+d\left(x_{m(p)}, x_{n(p)-1}\right) \\
d\left(x_{m}(p)-1, x_{n(p)-1}\right)<d\left(x_{m}(p)-1, x_{m}(p)\right)+\epsilon & 2.15 \\
\text { Hence } & 2.16 \\
\lim _{p \rightarrow \infty} \int_{0}^{d\left(x_{m}(p)-1, x_{n}(p)-1\right)} \xi(t) d t=\int_{0}^{\epsilon} \xi(t) d t & \\
\text { we get } &
\end{array}
$$

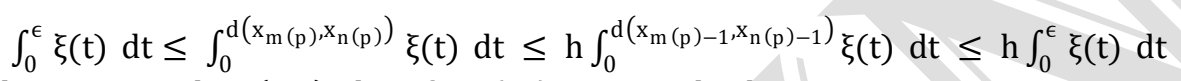

Which is contradiction, since $h \in(0,1)$. therefore $\left\{x_{n}\right\}$ is a Cauchy, hence converges to $\mathrm{z} \in \mathrm{X}$ from 2.9, we get

$$
\begin{array}{r}
\int_{0}^{d\left(f z, g x_{n}\right)} \xi(t) d t \leq \alpha \int_{0}^{\left[d(z, f z)+d\left(x_{n}, g x_{n}\right)\right]} \xi(t) d t+\beta \int_{0}^{\left[d\left(z, g x_{n}\right)+d\left(x_{n}, f z\right)\right]} \xi(t) d t \\
+\gamma \int_{0}^{\max \left\{d\left(z, g x_{n}\right), d\left(x_{n}, f z\right)\right\}} \xi(t) d t+\delta \int_{0}^{d\left(z, x_{n}\right)} \xi(t) d t
\end{array}
$$

Taking limit as $\mathrm{n} \rightarrow \infty$, we get

$$
\begin{aligned}
\int_{0}^{\mathrm{d}(\mathrm{fz}, \mathrm{z})} \xi(\mathrm{t}) \mathrm{dt} & \leq(\alpha+\beta+\gamma) \int_{0}^{\mathrm{d}(\mathrm{fz}, \mathrm{z})} \xi(\mathrm{t}) \mathrm{dt} \\
\text { as } 2 \alpha+2 \beta+2 \gamma+\delta & <1, \\
\int_{0}^{\mathrm{d}(\mathrm{fz}, \mathrm{z})} \xi(\mathrm{t}) \mathrm{dt} & =0
\end{aligned}
$$

Which, from 2.10 , implies that $\mathrm{d}(\mathrm{fz}, \mathrm{z})=0$ or $\mathrm{fz}=\mathrm{z}$. similarly it can be shown that $\mathrm{gz}=\mathrm{z}$. so $\mathrm{f}$ and $\mathrm{g}$ have a common fixed point $\mathrm{z} \in \mathrm{X}$. now we show that $\mathrm{z}$ is the unique common fixed point of $\mathrm{f}$ and $\mathrm{g}$. If not, then let $\mathrm{w}$ be another common fixed point of $f$ and $g$. Then from 2.9 we have,

$$
\begin{gathered}
\int_{0}^{\mathrm{d}(\mathrm{w}, \mathrm{z})} \xi(\mathrm{t}) \mathrm{dt} \leq \quad(2 \beta+\gamma+\delta) \int_{0}^{[\mathrm{d}(\mathrm{w}, \mathrm{gz})+\mathrm{d}(\mathrm{z}, \mathrm{fw})]} \xi(\mathrm{t}) \mathrm{dt} \\
\text { since } 2 \beta+\gamma+\delta<1 \text {, This implies that } \\
\int_{0}^{\mathrm{d}(\mathrm{w}, \mathrm{z})} \xi(\mathrm{t}) \mathrm{dt}=0
\end{gathered}
$$

Which from 2.10, implies that $\mathrm{d}(\mathrm{z}, \mathrm{w})=0$, and so the fixed point is unique.

Remark:

i. $\quad$ In Theorem 2.3 if we take $\mathrm{f}=\mathrm{g}$ then we get the result of Theorem 2.1.

References

1. S. Banach, Sur les oprations dans les ensembles abstraits et leur application aux quations intgrales, Fund. Math.3,(1922)133181 (French).

2. A. Branciari, A _xed point theorem for mappings satisfying a general contractive condition of integral type, Int.J.Math.Math.Sci, 29(2002), no.9, 531 - 536.

3. S.K.Chatterjea, Fixed point theorems, C.R.Acad.Bulgare Sci. 25(1972), 727-730.

4. K. Goebel and W. A. Kirk, Topiqs in Metric Fixed Point Theory, Combridge University Press, New York, 1990.

5. R. Kannan, Some results on xed points, Bull. Calcutta Math. Soc. , 60(1968), 71-76.

6. B.E. Rhoades, Two _xed point theorems for mappings satisfying a general contractive condition of integral type, International Journal of Mathematics and Mathematical Sciences, 63, (2003), 4007 - 4013.

7. B. E. Rhoades, A Comparison of Various De_nitions of Contractive Mappings, Trans. Amer. Math. Soc. 226 (1977), 257-290.

8. B. E. Rhoades, Contractive de_nitions revisited, Topological Methods in Nonlinear Functional Analysis (Toronto, Ont.,1982), Contemp. Math., 21, American Mathematical Society, Rhode Island, (1983), 189-203.

9. B. E. Rhoades, Contractive De_nitions, Nonlinear Analysis, World Science Publishing, Singapore, 1987, 513-526.

10. O. R. Smart, Fixed Point Theorems, Cambridge University Press, London, 1974.

11. T.Zamfrescu, Fixed point theorems in metric spaces, Arch.Math.(Basel) 23(1972), 292-298.

Acknowledgement: Author is thankful to MPCOST Bhopal (M.P) for the project "Application of fixed point theory in pure and applied mathematics" 